\title{
A psychological perspective towards understanding the objective and subjective gray zones in predatory publishing
}

\author{
Yuki Yamada' $^{1}$. Jaime A. Teixeira da Silva²
}

Accepted: 17 December 2021 / Published online: 13 January 2022

(c) The Author(s) 2022

\begin{abstract}
A continued lack of clarity persists because academics, policymakers, and other interested parties are unable to clearly define what is a "predatory" journal or publisher, and a potentially wide gray zone exists there. In this perspective, we argue that journals should be evaluated on a continuum, and not just in two shades, black and white. Since evaluations about what might constitute "predatory" are made by humans, the psychological decision-making system that determines them may induce biases. Considering such human psychological characteristics might shed light on the deterministic criteria that have been used, and continue to be used, to classify a journal or publisher as "predatory", and perhaps, bring additional clarity to this discussion. Better methods of journal evaluation can be obtained when the factors that polarize journal evaluations are identified. As one example, we need to move away from simply using whitelists and blacklists and educate individual researchers about how to evaluate journals. This paper serves as an educational tool by providing more clarity about the "gray" publishing zone, and argues that currently available qualitative and quantitative systems should be fused to deterministically appreciate the zonation of white, gray and black journals, so as to possibly reduce or eliminate the influence of cognitive or "perception" bias from the "predatory" publishing debate.
\end{abstract}

Keywords Academic and scholarly communication - Cognitive bias · Blacklists · Decision making $\cdot$ False discovery rate $\cdot$ Open access $\cdot$ Quality $\cdot$ Whitelists

Yuki Yamada

yamadayuk@gmail.com

Jaime A. Teixeira da Silva jaimetex@yahoo.com

1 Faculty of Arts and Science, Kyushu University, 744 Motooka, Nishi-ku, 819-0395 Fukuoka, Japan

2 Independent researcher, 3011-2 Ikenobe, 761-0799 Kagawa, Japan 


\section{Blacklisting and "predatory" publishing: a brief overview}

The topic of "predatory" publishing is not only highly topical, it constitutes a real issue in academic and scholarly publishing. For example, from 842 results at PubMed to date (December 15, 2021), 616 ( 73\%) were published in 2017-2021. ${ }^{1}$ Here, we briefly introduce the concept of 'predatory publishing', as characterized by some prominent papers on this topic, for readers to appreciate that while general concepts and characteristics are quite consistently known, definitions and solutions can vary widely. A highly-cited Nature commentary on this issue, published by Grudniewicz et al. (2019), describes a summit that was organized with a number of publishing specialists to formulate an agreed definition of predatory publishing using a modified Delphi survey. Although that summit resulted in a definition that was reached by consensus, it was reached exclusively by those who participated in it, so its generalizability needs to be carefully considered. The importance and difficulty of defining predatory publishing has also been discussed by others Aromataris and Stern 2020; Siler 2020; Teixeira da Silva 2020a). The major problem that has been discussed is that the degree and types of characteristics and practices in predatory publishing are numerous, complex, and intertwined, and even these include subjective elements. If it is difficult to define, should individuals select journals using a checklist of predatory publishing characteristics? A systematic review by Cukier et al. (2020) found 93 such checklists. Consequently, researchers might not know which checklists to trust, and may have trouble interpreting them if they show conflicting results.

Based on an understanding and characterization of predatory publishing, several measures have been proposed. For example, Manley (2019) addressed the legal case of OMICS, and clarified a number of points for publishers and editors related to the steps and measures required to avoid being regarded as engaging in predatory publishing (e.g., clarification of the payment scheme, installation of a dedicated submission system, specific description of the peer review system, and elimination of the use of misleading journal metrics) and for researchers to avoid being regarded as contributing to it (e.g., cautiousness about tempting submission invitations, declining poor or zero peer review, and not responding overzealously to requests to serve on editorial boards). Yet, the lack of national or international bodies to regulate and supervise such practices, and the apparent insufficient education of researchers, remain problematic. Thus, to help researchers easily identify and reject predatory journals, two prominent blacklists were established by Jeffrey Beall and Cabells. However, those listings have been criticized for their many shortcomings Dony et al. 2020; Teixeira da Silva and Tsigaris 2020; Tsigaris and Teixeira da Silva 2021a), and an alternative evaluation system has been proposed (Teixeira da Silva et al. 2021a). This will be discussed in more detail later in this paper.

\section{An introductory comment on the gray zone in blacklists and "predatory" publishing}

"Predatory" publishing can be found in open access (OA) publishers and/or journals, as well as in subscription-based journals (Olivarez et al. 2018). Despite an attempt to forge a uni-

${ }^{1}$ https://pubmed.ncbi.nlm.nih.gov/?term=predatory+publishing (raw results, which might also include false positives). 
fied definition for "predatory" publishing (Grudniewicz et al. 2019), false positives persist in publishing blacklists caused by low sensitivity and precision of selection criteria used for inclusion (Teixeira da Silva and Tsigaris 2020; Tsigaris and Teixeira da Silva 2021a). For example, a recent reevaluation of Beall's 55 blacklist criteria suggested that nine could be maintained, 24 should be eliminated, while the remaining 22 require corrections or modifications (Teixeira da Silva et al. 2022). These issues indicate the possibility that a gray zone

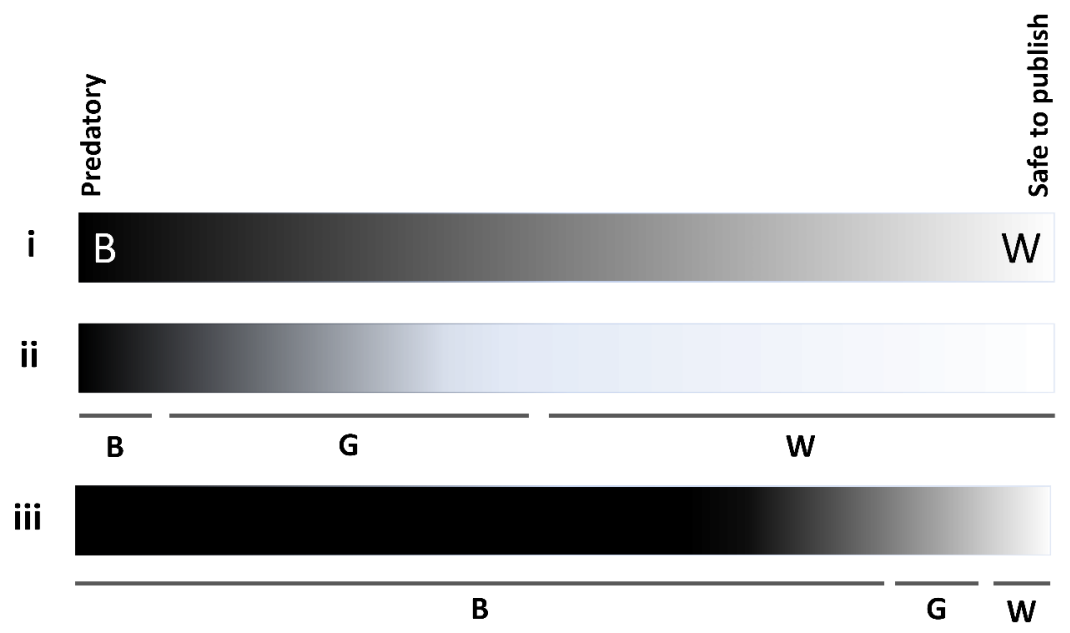

Fig. 1 Perception of the "predatory" nature of a journal (or publisher), or its safety, quality and reliability, might include absolutely clear positive (safe to publish in, or white) cases or absolutely clear negative (dangerous to publish in, or predatory) cases (black). Readers are cautioned that this is not merely an open access phenomenon, and can afflict any publishing model. In reality, there is a continuum of quality of academic journals, ranging from white to black, and endless shades of gray in between (case i). Gray represents some level of imperfection, and thus some risk to a career, reputation, etc. This continuum exists because each journal may have strengths and weaknesses that its competitors might not have. However, the limits between white, gray and white areas can depend on individual perceptions. Therefore, an individual with a high threshold for the negative evaluation of gray journals might not only select "safe" and whitelisted journals to publish in, but might also venture into the gray zone (case ii). This results in a wide range of journals that would, to such an individual, be considered "white", but also a range of gray journals to publish in that may carry some risks, but still be possible "acceptable" outlets for publication of their work. Even so, through a process of elimination, a narrow band of black (including predatory) journals remains. In contrast, in case iii, an individual with a low threshold for the negative evaluation of gray journals would select only "safe", whitelisted journals, and never, or rarely, venture into the gray zone of journals, and never into the black zone. This reduces the number of journals that would be considered safe to publish in (white) to a narrow belt. Similarly, the number of journals that are considered to be gray, i.e., that carry some risk but that are still possibly worthy of publication in, also lie in a narrow belt, while the black zone (i.e., unsafe to publish in, including actual or perceived predatory journals) expands widely. In cases ii and iii, the solid line under the black-to-white gradation (wide belt above solid line) of the continuum of black, gray, or white journals reflects that individual's perceived zone of black, gray, or white journals. Colors: B, black; G, gray; W, white 
of such journals and publishers exists, in terms of structural, managerial, and publishing operations (Siler 2020; Teixeira da Silva 2020b; Krawczyk and Kulczycki 2021). The abuse of such a gray zone allows individuals or groups to target - potentially unfairly — other academics merely based on the presence of an individual's publication on a blacklisted venue, but this is an unscholarly manner of characterizing the work of other academics (Teixeira da Silva and Kimotho 2021), unless unfair benefit can be clearly shown. Even though ample papers have been published on the topic of "predatory" publishing, no concrete solution that is specific to "predatory" publishers and/or journals exists yet, and current defense mechanisms that are based on ethical inquiry and post-publication peer-review or discovery (Yamada 2021) rely primarily on tools with generic advice that are applicable to any form of publishing, predatory or not.

Since any issue in everyday life is not a mere existence of a binary choice, nor a mere black or white selection, we are of the belief that a continuum of black- or white-leaning choices exist in the belt of gray "predatory" publishing. To try and better understand how to fortify means to detect and prevent "predatory" publishing, concepts that are artificially created by humans, we felt that it would be fitting to examine the influence of humans' psychological characteristics to assess an aspect as neither being black, nor white, but rather gray.

\section{Ambiguity may be negatively perceived by humans}

Human cognitive and emotional systems do not prefer to leave ambiguous things ambiguous (Yamada et al. 2012, 2013, 2014). One reason is presumably because treating the ambiguous as ambiguous is a demanding or disfluent task for human mental processing. Thus, if a journal is perceived as being neither black nor white, negative emotions or responses may arise simply because it is associated with the impression of being "gray" (i.e., uncategorized). This polarization of emotional valence is asymmetrical, biased in the negative direction, and hardly any cases are known of positive emotions arising in this context of "predatory" publishing. Therefore, journals that might lie in the gray zone, or lesser-known journals, are more likely to be treated with suspicion or negatively, and as "truly" predatory journals than traditional, well-known "respected" journals. This suspicion, combined with the growing discovery of failure in peer review-related quality control - as partly evidenced by a rise in retractions, and discovered through post-publication peer review-in traditionally perceived whitelisted and safe-to-publish-in journals, e.g. indexed on PubMed (Teixeira da Silva 2021a), a growing number of journals may fall into this zone (Fig. 1, case i). This possibility might be compounded by an increase in the variety of journals, so it may be increasingly common for journals to fail to receive the fair and due acknowledgment they deserve.

In ambiguous situations of a gray zone, the smoke detector principle (Nesse 2001), in which it is easier to cluster ambiguous cases with cases on the bad (i.e., predatory) side, seems to be at play because it is easier to deal with potentially harmful situations by placing them there. In other words, some might make a very cursory classification of journals in which all, except well-known journals, are predatory. Such individuals merely choose safe, whitelisted journals without having to think at all about what determines a predatory nature. Consequently, we believe that the threshold for the negative evaluation of gray journals is lowered, possibly causing the evaluation asymmetry to tend towards black (Fig. 1, case iii). For such individuals, due to their low or restrictive perceptive threshold, the world 
of publishing is populated heavily by "black" (including predatory) journals. Conversely, other individuals might appreciate that flawless journals (i.e., white) are rare, possibly even impossible to encounter, but also recognizing the risk of truly predatory journals and their existence, and might thus be more accommodating of a wider range of journals as being safe to publish in. Such individuals would likely embrace a wider zone of gray (Fig. 1, case ii) than case iii individuals in Fig. 1. For such individuals, due to their higher or less restrictive perceptive threshold, the world of publishing is populated heavily by gray or white journals, but also cognizant of "black" (including predatory) journals. Evidently, there is a wide range of individuals beyond this binary classification. Consequently, as we see it, a risk-averse academic (or a purely status quo-respecting one) would likely select a narrow zone of perceived white-categorized journals and more rarely gray-categorized journals in which to publish their work (Fig. 1, case iii). In contrast, a more liberal or accommodating (less risk averse) academic would likely have a wider zone of perceived white- and gray-categorized journals (or publishers) in their library of target journals (Fig. 1, case ii).

It is here that we expand slightly upon which individuals might fall into these categories (cases ii and iii) of perception. Experienced researchers who value their publishing venue and who are able to produce high-quality research, which may be proportional to their research funding, who are risk averse, and who are not concerned with quantitative output, would be likely candidates of case iii. In contrast, relative to such individuals, individuals with less research or publishing experience, such as early career researchers (ECRs), might be willing to take risks to advance their careers, which could involve the selection of journals of lower rank, of less credibility, and of poorer quality control, in doing so risking their reputation and careers, especially if critiqued by more radical elements of the antipredatory publishing movement. ECRs are very relevant to this topic since, more than most professional researchers, they are likely to be less exposed to information about the current state of predatory publishing. Moreover, they are more susceptible to the strong pressures associated with the "publish or perish" (Dyke 2019; Yamada 2019) culture, and are often motivated to publish many papers quickly, which can lead to poor submission choices if they are uninformed.

A publication derby for academic jobs is another confounding factor since the academic world is highly competitive (Yamada 2019) and the number of publications is still used to evaluate individual performance (Reinero 2019). Productivity in the first five years after the first publication can predict survival in the academic world (Milojević et al. 2018). In another report, the average number of papers published by those who obtained an assistant professor position in 2015-2016 Canadian cognitive psychology rose to twice that in 20062008 (Pennycook and Thompson 2018). In such a situation, one adaptive strategy would be to invalidate an adversary's publishing outlet by deliberately misclassifying it as "predatory", to bestow oneself an advantage over those with more publications. This process could be considered a novel form of "cancel" culture in academia (Teixeira da Silva 2021b). In this case, an attempt is made to cancel publication-related merits with blacklist-related hearsay. This process can result in gray journals being unfairly painted black. This could take place by an individual of class iii in Fig. 1 characterizing the publication curriculum of an individual of class ii, even if the individual in the latter class has papers published in journals classed as "white", even by the classification standards of the individual in the former class.

It is easy (but epistemologically wrong) for a journal to be evaluated as predatory if there is little information that can be positively evaluated as legitimate. However, if class ii 
individuals themselves have published papers in (not necessarily legitimate) gray journals, their own achievements can also fall into the predatory category, if publicly categorized as such by class iii individuals, for example on blogs, so the experimental objective to gain experience or diversify a publishing portfolio by publishing in gray journals thus becomes self-defeating because they might be unfairly categorized in public, or by their peers. Hence, we predict that those who publish papers exclusively in traditional and established journals to support their achievements, as suggested by Cappell (2015), will be more prone to this disparaging tendency and will, with unfounded confidence, call gray journals predatory, i.e., resulting in a class iii classification (Fig. 1). This happens if individuals rely solely on a journal's ranking or indexing legitimacy, which may be pseudo-quality factors (Teixeira da Silva 2021c), as the basis for evaluating it.
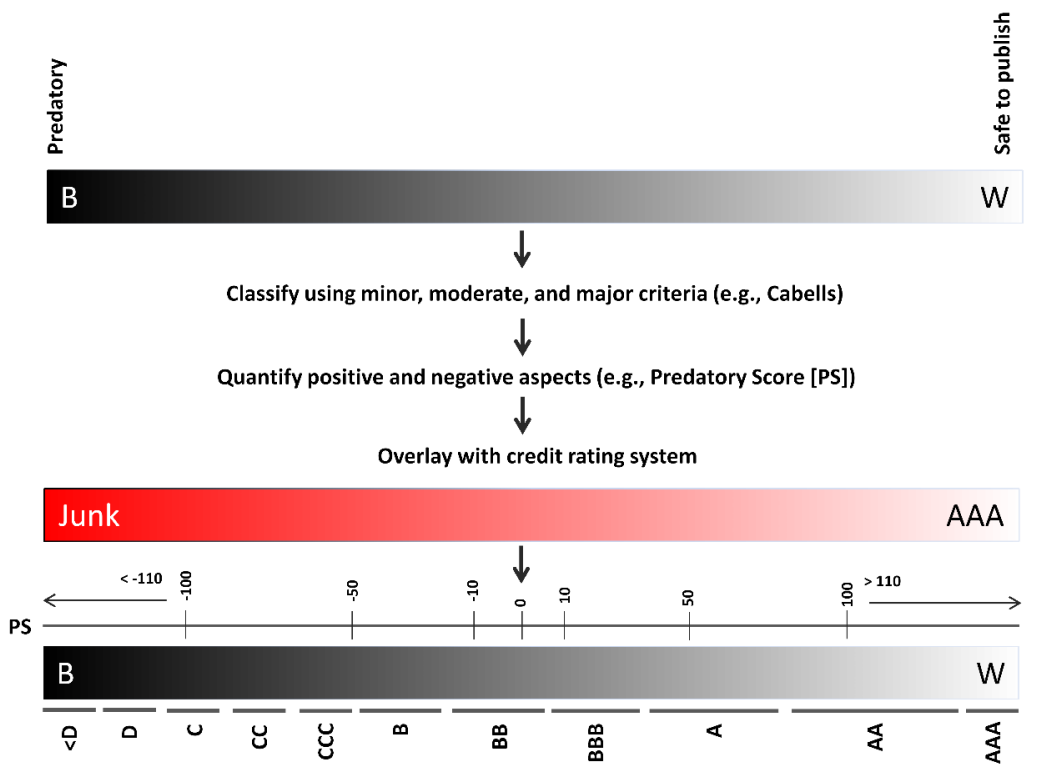

Fig. 2 What a system like the credit-like rating system adapted to predatory publishing (Teixeira da Silva et al., 2021) would attempt to do, is to assign quantitative values to risk, allowing white, gray and black zones to be more discernible, allowing a journal to receive a "rating". The ultimate objective of such a system would be to eliminate uncertainty caused by individual perceptions, as displayed by individuals of cases ii and iii in Fig. 1, in essence attempting to objectivize subjectivity. Consequently, artificially created "corrals" are superimposed on an indiscernible range of black, gray and white journals in order to appreciate classes (credit-like rating system), which themselves are defined using established criteria (e.g., those by Cabells), together with positive and negative publishing parameters that are scored by a relative weighting such as the Predatory Score (Teixeira da Silva 2013). Readers should note that Cabells' criteria, and thus blacklists (Predatory Reports) are imperfect (Dony et al. 2020) while the Predatory Score is now outdated and requires an upgrade. Although this represents a hypothetical road map forward to discerning "predatory" from unscholarly and scholarly journals, it might only be effective if: (1) it was constantly updated; (2) open to the public for free and independent verification; (3) approved by ethics and publishing-related organizations with a global reach and influence (COPE, ICMJE, etc.) 
Thus, gray journals are psychologically likely to be actively painted black (i.e., the issue of perception in Fig. 1). At present, the only types of solution that academics are providing is to apply quantitative indicators to reduce ambiguity, for example the Predatory Score (Teixeira da Silva 2013), or the accumulation of minor, medium and major faults in Cabells' Predatory Reports ${ }^{2}$, which have a high rate of false discovery (Dony et al. 2020). One way to acknowledge the existence of the gray zone, even when quantitative indicators are used, is by applying an overlay system akin to the credit-rating system (CRS) in place by financial institutions, rating journals, publishers, or others between AAA and "junk" (Teixeira da Silva et al. 2021). By doing so, at least in principle, the indistinguishable and poorly quantified gray zone can be more clearly differentiated from white and black zones when stratified by the CRS after having been evaluated by criteria (e.g., Cabells') and after positive and negative quality-related parameters have been quantified (e.g., using the Predatory Score) (Fig. 2).

\section{Behavioral characteristics behind submissions to predatorily gray journals}

In gray journals, it is often difficult for those who are not familiar with them to know whether peer review is actually taking place there or not, and as mentioned above, their reputation (e.g., on blogs or social media) might not be good. The "peer-reviewed" classification is usually not considered when achievements on journal papers are evaluated because peer review is automatically assumed in traditional, well-known whitelisted journals, but gray journals may sometimes be considered non-peer-reviewed, even if contrary to the fact. Therefore, submitting manuscripts to gray journals might not increase the number of apparent "peer-reviewed" papers. This means that there is little benefit for either the traditionlovers or number-lovers. Why, then, if knowledgeable of the risks, do some authors (class iii in Fig. 1) submit to such gray journals? Here, motivation and decision-making likely explain the behavior.

Considering the apparent changes in researchers' own reputation regarding the choice of journals to which they submit, decision-making theories in economics and psychology may be applied to their preferences. When submitting a manuscript to a journal, in situations where the credibility of the journal is uncertain (or gray), decisions can be made to maximize the researcher's own expected reputation in the scientific community, taking it as a utility (Edwards and Barron 1994; Savage 1954; von Neumann and Morgenstern 1944). Consequently, publication in a gray journal could be a factor of diversification or scholarly enrichment, provided that there is a sufficient mass of papers published in perceived white journals to disprove critics' unfounded theories. Or, if a researcher is subjectively weighing the probability of a change in their reputation as a result of submitting to a gray journal (Kahneman and Tversky 1979; Tversky and Kahneman 1992). However, the application of prospect theory to predatory publishing still requires a lot of work and careful thought prior to applying it to this field of research. The possible merits of publication in a journal, high reputation as well as prestige, financial reward, and tenure, are gains, while low reputation coming from the negative profile of the journal and high publication fees are losses although the latter are increasingly associated with whitelisted journals. In prospect theory, value is

${ }^{2}$ https://blog.cabells.com/category/predatory-reports/. 
relative, based on a reference point, which may vary depending on the situation and context. For example, for a researcher with no or few achievements, publication, even in a journal of a not particularly good reputation will be a great gain, while for a researcher with sufficient funds, the publication fee will not be much of a loss, with much of this money coming from public funding sources (Moher et al. 2017), and most likely not their private funds. Here, when evaluating gains and losses from the reference point of the latter category of researcher, loss aversion is supposed where losses outweigh gains. Thus, loss aversion may discourage researchers who have public research funds from submitting to gray and black (predatory) journals that are likely to generate stronger negative reputation. Consequently, an individual of class ii (Fig. 1) that publishes an abnormal mass of papers in the gray zone, with minimal productivity in journals in the white zone, will likely be perceived by an individual of class iii as favoring "low quality" or "predatory" publishing outlets.

Alternatively, findings in areas related to risk analysis, such as risk compensation behavior (Peltzman 1975; Wilde 1982), are also worth considering. Risk compensation is the tendency to take an extra risky action when the risk is perceived to be reduced, and vice versa. For example, if a gray journal is found not to be on a blacklist (i.e., if it is perceived to be safer or have lower risk), risky submissions to a non-blacklisted journal may be more likely to occur, even if there are other suspicious aspects that are ignored. If this is the case, then overconfidence in inappropriate blacklists may have rather negative consequences for the submitters themselves.

Practically speaking, this has serious consequences. For example, if a blacklist employs a lax (or opaque) set of inclusive criteria, and an unrelated whitelist also employs a lax (or opaque) set of inclusive criteria, there is a risk of finding an overlap between blacklisted and whitelisted journals and publishers (Teixeira da Silva 2020b). The extreme risk and danger to academic research is when the claim that the blacklisted entity-established according to blacklist criteria "a", and thus labeled as "predatory"-is extended to a whitelisted entity - established according to whitelist criteria "b", and thus labeled as "safe" — in order to ridicule or otherwise reduce the academic or scholarly validity of the whitelisted entity. An example of this risk lies in the now-retracted Macháček and Srholec (2021) paper, as argued through the prism of "national propensities" (Mills and Bell 2021).

All of the above is an individual psychological matter. We need to extend the discussion to the social scale (e.g., rumor mongering, collective sympathy, in-group favors, collective decision-making, etc.), although this is beyond the scope of the present paper. It is important that further empirical evidence is accumulated on each topic.

\section{Consequences of clustering gray entities as blacklisted "predatory" publishing}

The first obvious outcome, or consequence, of clustering all entities that violate anything from one to multiple "criteria" for being considered a "predatory" journal or publisher is that it fosters a culture of unfairness and stigmatization. When such a classification is then extended to an entire particular racial or cultural group, actual or possible racism results if the entire academic body of that nation is characterized in the same negative manner without due and balanced or fair consideration of the meritorious positive aspects of the same academic body (Teixeira da Silva and Kimotho 2021). This is what happened when a pseud- 
onymous (fake) "author", Truth (2012) made unsupported claims about specific cultures and even groups of academics of specific religious affiliations (e.g., Muslims), to classify their publishing behavior or choice of publishing venue as "predatory". Almost a decade later, the same attitude and risk continues to be propagated, as a modified philosophy, "national propensities" (Macháček and Srholec 2021), although that paper has now been retracted. This allows academics to reflect more carefully on applying a biased perceptive position (e.g., classes ii or iii in Fig. 1) to individuals, groups or cultures that may have different perspectives.

The second risk, as alluded to briefly above, is an attempt to use the "predatory" label to try and invalidate adversaries' work based on publishing outlets, thereby gaining reputational superiority due to misclassification and mischaracterization or competitors. A recent high-profile case (Derek Pyne) attempted to denigrate some papers and publication achievements of more than a dozen of his competitors (i.e., tenured staff in the same school) by attaching a negative label to them (i.e., the papers and, by association, the researchers who published them) by claiming that their choice of publishing venues were "predatory" because they had been published in venues that were blacklisted by Beall (Pyne 2017). This mischaracterization negatively affected all researchers at that university, by association. In addition, Pyne (2017) falsely claimed, as revealed by reproducibility issues and flaws related to that paper (Tsigaris and Teixeira da Silva 2019, 2020b), that his competitors benefited, supposedly by publishing quickly in journals and with publishers on Beall's blacklists, satisfying their research requirement, and then taking teaching overloads to increase their salary. Even though any reader will believe that such rewards exist, merely by reading the title and abstract of the paper, such rewards were not supported by the evidence provided in that research (Tsigaris 2019). This obscurely unsupported hypothesis of rewards is unlike the many institutions or countries in which the choice of publication venue, and its rank (e.g., impact factor, CiteScore, etc.) or whitelisted index (Scopus, PubMed, Web of Science, etc.), tends to be explicitly financially rewarded. Despite this, several misled academics who fell for the hype and spin of that paper, or employed its erroneous findings to support false hypotheses of rewards for "predatory" publications, continue to cite the Pyne (2017) paper indiscriminately (Tsigaris and Teixeira da Silva 2021b). Rewards, in the form of citations to erroneous research, can thus be reaped based on research that erroneously classifies "predatory" publishing and fails to see, or appreciate, the indistinct and wide gray zone in academic publishing (Fig. 1, case i).

The third risk is the potential to propagate fallacy at various levels based on unreliable blacklists, such as those by Beall, or even Cabells (Dony et al. 2020). Claiming that journal or publisher "A" is "predatory" when in fact it might not be, based exclusively on the claim that it exists on a blacklist, reputationally harms all academics who may have published in the same journal, or with the same publisher. The novel concept of "mass slander" then arises, in which all academics of a common entity (university, country, etc.) within a particular cluster (blacklisted journal or publisher) are judged unfairly, or inaccurately, based on the same erroneous value systems, usually by class ii (Fig. 1) individuals. Moreover, as mentioned above, trying to evaluate a journal solely on the basis of whether or not it appears on such lists can create the danger of thoughtlessly submitting to gray journals that might be likely, potentially, or truly black (i.e., predatory).

To avoid such risks in an entire field, human cognitive processes need to be taken more into account. The human factors approach, which considers psychological and physical fac- 
tors separately, has been discussed in various fields where risk management is particularly important, and therein, an understanding of cognitive processing is emphasized (e.g., Henriksen and Brady 2013; Dror 2020). Thus, various factors (e.g., blacklists and rumors on unofficial blogs or social media), one example in Teixeira da Silva (2019), that lead to inappropriate and incorrect psychological clustering, need to be eliminated. On the other hand, rich information relevant to journal selection should be provided in highly accessible spaces (i.e., the information should not be paid for, e.g., Cabells' Predatory Reports), and rating systems (Teixeira da Silva et al. 2021) and educational curricula should be developed to prevent the inappropriate clustering and biased discriminatory evaluations based on nationality and religion.

\section{Limitations and future needs}

Unfortunately, a definitive solution to "predatory" publishing has not been found, in part because academia has not been able to clearly differentiate predatory from non-predatory entities, i.e., it has been unable to parse the gray zone, in some cases preferring to employ the term "questionable" to encompass both "bad-faith outlets from low-quality ones" (Kulczycki et al. 2021). In part, this has been due to the creation of blacklists with insufficiently clear criteria, or the blacklisting or whitelisting of journals or publishers based on insufficiently robust criteria, or due to the failure to appreciate, and acknowledge, the existence of the wide gray zone of quality in academic standards and scholarly behavior in journals or publishers. Compounding these issues, the perceived black, gray, or white zones will depend on individual experiences, perceptions, and biases. In addition, the psychological characteristics of manuscript submission depend on the extent to which a researcher knows about "predatory" journals and is aware of their existence. Here, however, we feel that there is a body of literature that might in fact be providing erroneous and thus bad advice, essentially misinformation, a topic that is currently being explored through post-publication peer review. These psychological or ideological differences can cause serious misinformation if such "advice" papers are published by individuals of classes ii and iii (Fig. 1), or if they are appointed as "quality" sentinels for academia, such as in ethics groups, policy makers, influencers, or media outlets.

Since ECRs are the "future" of academia, solutions are needed to ensure that their research is published properly and in safe venues that are reputable and that embrace measures that constantly reassess quality control (Teixeira da Silva 2021d). To achieve this, educational measures for ECRs need to be further strengthened in the future, since the coping strategies we have discussed here may not be sufficiently effective if they have insufficient knowledge about predatory publishing.

Acknowledgements The authors thank the positive critique and input offered by Prof. Panagiotis Tsigaris (Department of Economics, Thompson Rivers University, Canada) on an early revised version of the paper.

Authors' contributions The authors contributed equally to the intellectual discussion underlying this paper, literature exploration, writing, reviews, and editing, and accept responsibility for the content of this paper.

Conflict of interest and funding The authors declare no conflicts of interest of relevance to this topic. Although this study and project was not funded, YY was funded by JSPS KAKENHI (16H03079, 17H00875, $18 \mathrm{~K} 12015,20 \mathrm{H} 04581$, and 21H03784). JTS is not funded. 
Open Access This article is licensed under a Creative Commons Attribution 4.0 International License, which permits use, sharing, adaptation, distribution and reproduction in any medium or format, as long as you give appropriate credit to the original author(s) and the source, provide a link to the Creative Commons licence, and indicate if changes were made. The images or other third party material in this article are included in the article's Creative Commons licence, unless indicated otherwise in a credit line to the material. If material is not included in the article's Creative Commons licence and your intended use is not permitted by statutory regulation or exceeds the permitted use, you will need to obtain permission directly from the copyright holder. To view a copy of this licence, visit http://creativecommons.org/licenses/by/4.0/.

\section{References}

Aromataris, E., Stern, C.: Supporting a definition of predatory publishing. BMC Med. 18, 125 (2020). https:// doi.org/10.1186/s12916-020-01599-6

Cappell, M.S.: List predatory journal publications separately from genuine scholarly publications as standard for CVs. BMJ. 350, h2470 (2015). https://doi.org/10.1136/bmj.h2470

Cukier, S., Helal, L., Rice, D.B., Pupkaite, J., Ahmadzai, N., Wilson, M., Skidmore, B., Lalu, M.M., Moher, D.: Checklists to detect potential predatory biomedical journals: A systematic review. BMC Med. 18(1), 104 (2020). https://doi.org/10.1186/s12916-020-01566-1

Dror, I.: Cognitive and human factors in expert decision making: Six fallacies and the eight sources of bias. Anal. Chem. 92(12), 7998-8004 (2020). https://doi.org/10.1021/acs.analchem.0c00704

Dony, C., Raskinet, M., Renaville, F., Simon, S., Thirion, P.: How reliable and useful is Cabell's blacklist? A data-driven analysis. LIBER Q. 30(1), 1-38 (2020). https://doi.org/10.18352/lq.10339

Dyke, G.: Does the early career 'publish or perish' myth represent an opportunity for the publishing industry? Learn. Publish. 32(1), 90-94 (2019). https://doi.org/10.1002/leap.1217

Edwards, W., Barron, F.H.: SMARTS and SMARTER: Improved simple methods for multiattribute utility measurement. Organ. Behav. Hum. Decis. Process. 60, 306-325 (1994). https://doi.org/10.1006/ obhd.1994.1087

Grudniewicz, A., Moher, D., Cobey, K.D., Bryson, G.L., Cukier, S., Allen, K., Ardern, C., Balcom, L., Barros, T., Berger, M., Ciro, J.B., Cugusi, L., Donaldson, M.R., Egger, M., Graham, I.D., Hodgkinson, M., Khan, K.M., Mabizela, M., Manca, A., Milzow, K., Mouton, J., Muchenje, M., Olijhoek, T., Ommaya, A., Patwardhan, B., Poff, D., Proulx, L., Rodger, M., Severin, A., Strinzel, M., Sylos-Labini, M., Tamblyn, R., van Niekerk, M., Wicherts, J.M., Lalu, M.M.: Predatory journals: no definition, no defence. Nature. 576(7786), 210-212 (2019). https://doi.org/10.1038/d41586-019-03759-y

Henriksen, K., Brady, J.: The pursuit of better diagnostic performance: A human factors perspective. BMJ Qual. Saf. 22(Suppl 2), ii1-ii5 (2013). https://doi.org/10.1136/bmjqs-2013-001827

Kahneman, D., Tversky, A.: Prospect theory: An analysis of decision under risk. Econometrica. 47(2), 263291 (1979). https://doi.org/10.2307/1914185

Krawczyk, F., Kulczycki, E.: How is open access accused of being predatory? The impact of Beall's lists of predatory journals on academic publishing. J. Acad. Librariansh. 47(2), 102271 (2021). https://doi. org/10.1016/j.acalib.2020.102271

Kulczycki, E., Hołowiecki, M., Taşkın, Z., Krawczyk, F.: Citation patterns between impact-factor and questionable journals. Scientometrics. 126(10), 8541-8560 (2021). https://doi.org/10.1007/ s11192-021-04121-8

Macháček, V., Srholec, M.: Predatory publications in Scopus: evidence on cross-country differences. Scientometrics, 126(3), 1897-1921 (2021). https://doi.org/10.1007/s11192-020-03852-4 retraction: https:// doi.org/10.1007/s11192-021-04149-w

Manley, S.: Predatory journals on trial: Allegations, responses, and lessons for scholarly publishing from FTC v. OMICS. J. Sch. Publ. 50(3), 183-200 (2019). https://doi.org/10.3138/jsp.50.3.02

Mills, D., Bell, K.: National propensities? Scientometrics. (2021 in press). https://doi.org/10.1007/s11192021-04014-w corrigendum: https://doi.org/10.1007/s11192-021-04070-2

Milojević, S., Radicchi, F., Walsh, J.P.: Changing demographics of scientific careers: The rise of the temporary workforce. Proc. Natl. Acad. Sci. U.S.A. 115(50), 12616-12623 (2018). https://doi.org/10.1073/ pnas. 1800478115

Moher, D., Shamseer, L., Cobey, K.D., Lalu, M.M., Galipeau, J., Avey, M.T., Ahmadzai, N., Alabousi, M., Barbeau, P., Beck, A., Daniel, R., Frank, R., Ghannad, M., Hamel, C., Hersi, M., Hutton, B., Isupov, I., McGrath, T.A., McInnes, M., Page, M.J., ... Ziai, H.: Stop this waste of people, animals and money. Nature. 549(7670), 23-25 (2017). https://doi.org/10.1038/549023a 
Nesse, R.M.: The smoke detector principle. Natural selection and the regulation of defensive responses. Ann. N. Y. Acad. Sci. 935, 75-85 (2001). https://doi.org/10.1111/j.1749-6632.2001.tb03472.x

Olivarez, J.D., Bales, S., Sare, L., vanDuinkerken, W.: Format aside: applying Beall's criteria to assess the predatory nature of both OA and non-OA library and information science journals. Coll. Res. Libr. 79(1), 52-67 (2018). https://doi.org/10.5860/crl.79.1.52

Pennycook, G., Thompson, V.A.: An analysis of the Canadian cognitive psychology job market (2006-2016). Can. J. Exp. Psychol. 72(2), 71-80 (2018). https://doi.org/10.1037/cep0000149

Peltzman, S.: The effects of automobile safety regulation. J. Polit. Econ. 83(4), 677-725 (1975). https://doi. org/10.1086/260352

Pyne, D.: The rewards of predatory publishing at a small business school. J. Sch. Publ. 48(3), 137-160 (2017). https://doi.org/10.3138/jsp.48.3.137

Reinero, D.A.:The path to professorship by the numbers and why mentorship matters. Behavioural and Social Sciences at Nature Research. (2019, October 23). https://socialsciences.nature.com/ posts/55118-the-path-to-professorship-by-the-numbers-and-why-mentorship-matters

Savage, L.J.: The foundations of statistics. John Wiley \& Sons (1954)

Siler, K.: Demarcating spectrum of predatory publishing: economic and institutional sources of academic legitimacy. J. Assoc. Inf. Sci. Technol. 71(11), 1386-1401 (2020). https://doi.org/10.1002/asi.24339

Teixeira da Silva, J.A.:Predatory publishing: a quantitative assessment, the Predatory Score. The Asian and Australasian Journal of Plant Science and Biotechnology, 7(Special Issue 1), 21-34 (2013)

Teixeira da Silva, J.A.: Leonid Schneider calls Springer Nature's Science and Engineering Ethics predatory, without proof. J. Advocacy Res. Edu. 6(1), 5-14 (2019)

Teixeira da Silva, J.A.: The ICMJE recommendations: challenges in fortifying publishing integrity. Ir. J. Med. Sci. 189(4), 1179-1181 (2020a). https://doi.org/10.1007/s11845-020-02227-1

Teixeira da Silva, J.A.: Is there a clear division between predatory and low-quality journals and publishers? J. R. Coll. Physicians Edinb. 50(4), 458-459 (2020b). https://doi.org/10.4997/JRCPE.2020.303

Teixeira da Silva, J.A.: Is the validity, credibility and reliability of literature indexed in PubMed at risk? Med J. Armed Forces India. (2021a in press). https://doi.org/10.1016/j.mjafi.2021.03.009

Teixeira da Silva, J.A.: How to shape academic freedom in the digital age? Are the retractions of opinionated papers a prelude to "cancel culture" in academia? Curr. Opin. Behav. Sci. 2, 100035 (2021b). https:// doi.org/10.1016/j.crbeha.2021.100035

Teixeira da Silva, J.A.: Citations and gamed metrics: academic integrity lost. Acad. Quest. 34(1), 96-99 (2021c). https://doi.org/10.51845/34s.1.18

Teixeira da Silva, J.A.: Challenges that early career researchers face in academic research and publishing: pre- and post-COVID-19 perspectives. Exchanges. 9(1), 77-106 (2021d). https://doi.org/10.31273/eirj. v9i1.882

Teixeira da Silva, J.A., Dunleavy, D.J., Moradzadeh, M., Eykens, J.: A credit-like rating system to determine the legitimacy of journals and publishers. Scientometrics. 126(10), 8589-8616 (2021). https://doi. org/10.1007/s11192-021-04118-3

Teixeira da Silva, J.A., Kimotho, S.G.: Signs of divisiveness, discrimination and stigmatization caused by Jeffrey Beall's “predatory" open access publishing blacklists and philosophy. J. Acad. Librariansh. (2021 in press). https://doi.org/10.1016/j.acalib.2021.102418

Teixeira da Silva, J.A., Moradzadeh, M., Adjei, K.O.K., Owusu-Ansah, C.M., Balehegn, M., Faúndez, E.I., Janodia, M.D., Al-Khatib, A.: An integrated paradigm shift to deal with "predatory" publishing. J. Acad. Librariansh. 48(1), 102481 (2022). https://doi.org/10.1016/j.acalib.2021.102481

Teixeira da Silva, J.A., Tsigaris, P.: Issues with criteria to evaluate blacklists: An epidemiological approach. J. Acad. Librariansh. 46(1), 102070 (2020). https://doi.org/10.1016/j.acalib.2019.102070

Truth, F.: Pay big to publish fast: academic journal rackets. J. Crit. Educ. Policy Stud. 10(2), 54-105 (2012)

Tsigaris, P.: Letter to the Editors. J. Sch. Publ. 50(2), 139-142 (2019). https://doi.org/10.3138/jsp.50.2.05

Tsigaris, P., Teixeira da Silva, J.A.: Did the research faculty at a small Canadian business school publish in "predatory" venues? This depends on the publishing blacklist. Publications 7(2), 35 (2019). https://doi. org/10.3390/publications7020035

Tsigaris, P., Teixeira da Silva, J.A.: Reproducibility issues with correlating Beall-listed publications and research awards at a small Canadian business school. Scientometrics 123(1), 143-157 (2020). https:// doi.org/10.1007/s11192-020-03353-4

Tsigaris, P., Teixeira da Silva, J.A.: Why blacklists are not reliable: A theoretical framework. J. Acad. Librariansh. 47(1), 102266 (2021a). https://doi.org/10.1016/j.acalib.2020.102266

Tsigaris, P., Teixeira da Silva, J.A.: Bibliometric analysis of a controversial paper on predatory publishing. Perform. Meas. Metr. 22(1), 39-47 (2021b). https://doi.org/10.1108/PMM-03-2020-0015

Tversky, A., Kahneman, D.: Advances in prospect theory: Cumulative representation of uncertainty. J. Risk. Uncertain. 5, 297-323 (1992). https://doi.org/10.1007/BF00122574 
von Neumann, J., Morgenstern, O.: Theory of Games and Economic Behavior. Princeton University Press (1944)

Wilde, G.J.: The theory of risk homeostasis: Implications for safety and health. Risk Anal. 2(4), 209-225 (1982). https://doi.org/10.1111/j.1539-6924.1982.tb01384.x

Yamada, Y.: Publish but perish regardless in Japan. Nat. Hum. Behav. 3, 1035 (2019). https://doi.org/10.1038/ s41562-019-0729-9

Yamada, Y.: How to protect the credibility of articles published in predatory journals. Publications. 9(1), 4 (2021). https://doi.org/10.3390/publications9010004

Yamada, Y., Kawabe, T., Ihaya, K.: Can you eat it? A link between categorization difficulty and food likability. Adv. Cogn. Psychol. 8, 248-254 (2012). https://doi.org/10.2478/v10053-008-0120-2

Yamada, Y., Kawabe, T., Ihaya, K.: Categorization difficulty is associated with negative evaluation in the "uncanny valley" phenomenon. Jpn. Psychol. Res. 55, 20-32 (2013). https://doi. org/10.1111/j.1468-5884.2012.00538.x

Yamada, Y., Sasaki, K., Kunieda, S., Wada, Y.: Scents boost preference for novel fruits. Appetite. 81, 102107 (2014). https://doi.org/10.1016/j.appet.2014.06.006

Publisher's Note Springer Nature remains neutral with regard to jurisdictional claims in published maps and institutional affiliations. 